\title{
Assessing the Graham's Formula for Stock Selection: Too Good to Be True?
}

\author{
Jason Lin', Jane Sung ${ }^{2}$ \\ ${ }^{1}$ Department of Business Administration, Truman State University, Kirksville, USA \\ ${ }^{2}$ Department of Economics, Truman State University, Kirksville, USA \\ Email: jlin@truman.edu
}

Received October 2013

\begin{abstract}
Benjamin Graham offered a straightforward and simple formula to evaluate stocks' intrinsic value. Many regard the Graham Formula is a very simplistic way of measuring an individual company's intrinsic value. Graham and Warren Buffet however felt that the simplicity of the model allowed them to quickly and accurately identify undervalued companies, and stay away from overvalued ones. In this paper, we wanted to explore the effectiveness of the Graham's formula. We wanted to see if using the Graham's formula, investors can achieve excess returns above the market over a period of 17 years.
\end{abstract}

\section{Keywords}

\section{Graham's Formula; Intrinsic Value}

\section{Introduction}

Many regard the Graham Formula is a very simplistic way of measuring an individual company's intrinsic value. Graham and Warren Buffet however felt that the simplicity of the model allowed them to quickly and accurately identify undervalued companies, and stay away from overvalued ones. They understood that other strategies could produce excess returns, but that this strategy allowed them to do so with less risk. The formula was originally developed by economist Benjamin Graham in 1962 and was further revised by Mr. Graham in 1974 [1]. The formula requires two company specific inputs and one systematic input. The two company specific inputs are the company's earnings per share for the past twelve months and the company's long-term earnings growth estimate. The one systematic input is the yield on AAA corporate bonds. This allows the investor to take into account economic conditions that change the risk premium for low risk bonds, and the specific earnings and the growth of these earnings. Although usually described as the father of value investing, the formula can more accurately be described as G.A.R.P. or growth at a reasonable price because the higher the growth the more value the formula attributes to the stock.

While many view this method as far too simplistic to predict minor fluctuations in the market, the Graham Formula has been proven to be extremely useful in analyzing the stock market crash in October 1987. A study in the May-June 1988 Harvard Business Review titled “The Smart Crash of October 19"th", the authors of the study, 
Arbel, Carvell, and Postnieks, (1988) [2] showed how remarkably well the Graham formula worked. The study showed that nearly all the stocks on the market were overvalued on October 1, 1987, using the Relative Graham Value (intrinsic value using Graham Formula divided by the current stock price). During the period preceding the crash many in the investing community talked about how the Graham formula was outdated and inconsistent with rational prices of the day. However, when the markets closed on October 19, 1987, the Relative Graham Value was remarkably close to one for a large majority of stocks listed on the exchange (Arbel, 1988). [2] The authors believed that instead of a burst of irrationality, the crash was a return to rationality when investors realized they couldn't make profits by buying overvalued securities and selling them at a higher price because eventually no one would pay that amount. During this period, the Graham formula was very accurate in predicting stock prices and the authors believe this is evidence that the Graham formula is a good tool for assigning value to a company's stock.

The authors of the study hypothesized that as the crash was happening, many investors went back to a fundamental approach of stock valuation, rather than the irrational exuberance that typified the 1980s stock market. This was made clear by the fact that a valuation technique such as the Graham Formula was so useful in explaining why stocks fell to the level they did. If the Graham formula was a good predictor of stock price, it would work best when markets were rational and there was no mispricing due to an over exuberance as the authors believed there was preceding the crash.

In this paper, we wanted to explore the effectiveness of the Graham's formula. We wanted to see if using the Graham's formula, investors can achieve excess returns above the market over a period of 17 years.

\section{Literature Review}

The Graham formula is a product of the 1930's market crash, and coming from those uncertain times, its goal was to "stand the test of the ever enigmatic future " as Graham himself mentioned in the preface to his book "Security Analyst" [3]. Graham developed his theory and elaborated on it in two of his most famous books, "Security Analyst" and "The Intelligent Investor". The main concept behind the formula is the belief that companies have an intrinsic value which the market doesn't necessarily reflect in their stock prices. The intrinsic value includes both tangible and intangible parts of the company and its determination is based on fundamental analysis rather than the stock price. Investor shouldn't be too concerned with the short term fluctuations in stock price, rather they should focus on the long run in order to achieve excess returns.

Benjamin Graham offered a straightforward and simple formula to evaluate stocks' intrinsic value. The formula consists of four inputs: current earnings per share, projected growth, the underlying appropriate earnings yield and interest expectations based on the AAA corporate bonds. This simplicity of the formula is in stark contrast to today's complex models, and perhaps because of its simplicity, it's often ignored in valuation methods and dismissed as too naive.

$$
\text { Value }=\frac{E P S \times(8.5+2 g) \times 4.4}{Y}
$$

Using Graham's formula, a Relative Graham Value (RGV) is calculated by dividing the stock's intrinsic value by its current price [2]. It can be used to analyze whether a stock is undervalued or overvalued. If the RGV is above one, according to this theory the stock is undervalued and thus a good buy. On the contrary, if the RGV is below one the stock is overvalued and thus a good sell. The idea behind the Graham's formula however, goes against developments in more recent financial theory, most notably the efficient market hypothesis. Whereas the followers of the efficient market hypothesis believe that the market incorporates any new information into the stock prices, proponents of Graham argue that this is not the case. Using the Graham's formula, they believe that stocks can be underpriced and overpriced by the market and as such, there exist opportunities for returns in excess of the market. Most notable investors that follow Benjamin Graham's philosophy are Warren Buffett, John Bogle and Mario Gabelli. The ideas of Graham are not mainstream however, and as stated earlier, many dismiss the formula at too simple and not sophisticated enough.

\section{Data and Methodology}

To assess the predictive value of Graham's formula, we used the stock price and EPS of the 30 Blue Chip com- 
panies that compose the Dow Jones index [4]. The rule that we followed was that if the stock reached a RGV over our predetermined criterion, it would be bought and if the RGV went below it, it would be sold. Several assumptions were used in finding the input values for the Graham formulas:

1) The time period used in the comparison went from 1997 until 2013.

2) Diluted EPS of the 30 blue chip stocks was used.

3) Growth rate used was the annualized growth in EPS over the previous five years; to prevent negative stock prices, we limited the growth factor to $-4.25 \%$ which would make $8.5+2 \mathrm{~g}=0$.

4) For corporate bond rates, we used annualized yield based on monthly bond yields.

5) Stock prices and Dow Jones data were taken from the adjusted close on June $30^{\text {th }}$ each year or the nearest previous trading day; furthermore stocks were adjusted for stock splits.

6) We checked to see if we needed to buy or sell stocks only on June $30^{\text {th }}$.

7) As the Dow Jones has changed composition, we followed suit; we only invested in stocks that were in the composite as of June $30^{\text {th }}$ and divested of stocks that left the composite.

Furthermore, our analysis was split in two separate trials with RGV levels of 1.25 and 1.50 respectively. We bought the stock when its RGV was above the level and sold it when the RGV went below one. The portfolio was composed of equal market values of every stock invested in at the beginning of each year. Finally, for the purpose of this portfolio we liquidated our positions in 2013 regardless of the RGV.

We attempted to remove all sources of survivorship bias by using the current Dow Jones component companies for each year. We were able to find most of the companies still exist in one form or another but two stocks' data were hard to come by, AT \& T before the merger with SBC and Union Carbide. In both of these cases, the company became a wholly owned subsidiary of another company and there is no currently traded stock that reflects their historical prices.

\section{Results}

Our findings related to Graham's formula's predictive power are quite remarkable. As stated before, we wanted to know whether or not Graham's formula could be used to achieve excess returns above the market as an investor. Benjamin Graham was a proponent of taking considerable margin of safety in investment decisions to allow for error in analysis and to provide for a stronger argument in favor of the same investment decision (Graham, 2006) [1]. For this reason, we assumed a position in the companies with RGV's greater than one at two different margins of safety levels. An RGV above one suggests that the company is currently underpriced in the market and should be bought, whereas an RGV below 1 suggests that the company is overvalued. With this in mind and to test the strength of Graham's formula, we assumed a portfolio that purchased into companies whenever their RGV was above one by a margin of safety of $25 \%$ and $50 \%$ (two separate trials) and sold the company's securities whenever their RGV fell below one. We felt that purchasing and selling at these respective levels was the best way to test the predictive power of Graham's formula because that is what it implied-an RGV above one means the company's stock is undervalued and an RGV below one means the company is overvalued. Again, what we found was pretty impressive.

If, starting before 1997, you selected companies to invest in based solely on Graham's formula and held the criteria that the companies must be trading at an RGV below one with a margin of safety of at $25 \%$, you would have outperformed the Dow Jones Industrial Average in every year from 1997 to 2013 except for three years (1998, 2003, and 2011). The following Table 1 shows our results with the first column being how much excess return Graham's formula was able to generate above the Dow Jones Industrial Average each year. The second column is measuring year over year, how much did the Graham's over perform the market on a cumulative basis. In the years that Graham's formula underperformed the market, it only underperformed marginally. When Graham's formula over performed the market, however, it did so substantially as we can see by 2013, the cumulative over performance by Graham's formula was $119.44 \%$ over the seventeen years.

For the portfolio where we took considerably more margin of safety at $50 \%$, the results were even better. Graham's formula performed better than the DJIA in every single year. The second half of the Table 1 summarizes our results for holding this portfolio. This phenomenal track record leads to the cumulative return over the DJIA in our second portfolio to be substantially higher, almost double that of our first portfolio.

If we look at the returns as a year over year compounded effect on total returns, we can see the difference in a measurable dollar amount. From 1997 to 2013, the Dow Jones Industrial Average went from 7672 to 14,975. If 
Table 1. Summery of portfolio returns.

\begin{tabular}{lcccc}
\hline & \multicolumn{2}{c}{ Case I } & \multicolumn{2}{c}{ Case II } \\
\cline { 2 - 5 } Buy at RGV >= 1.25 & \multicolumn{2}{c}{ Buy at RGV >= $\mathbf{. 5}$} \\
\cline { 2 - 5 } $\mathbf{1 9 9 8}$ & Return over DJIA & Cumulative Return & Return over DJIA & Cumulative Return \\
$\mathbf{1 9 9 9}$ & $-1.57 \%$ & $-1.57 \%$ & $0.21 \%$ & $0.21 \%$ \\
$\mathbf{2 0 0 0}$ & $4.95 \%$ & $3.30 \%$ & $5.05 \%$ & $5.27 \%$ \\
$\mathbf{2 0 0 1}$ & $6.15 \%$ & $9.66 \%$ & $6.15 \%$ & $11.74 \%$ \\
$\mathbf{2 0 0 2}$ & $19.95 \%$ & $31.53 \%$ & $22.05 \%$ & $36.38 \%$ \\
$\mathbf{2 0 0 3}$ & $2.95 \%$ & $35.41 \%$ & $4.91 \%$ & $43.08 \%$ \\
$\mathbf{2 0 0 4}$ & $-1.90 \%$ & $32.84 \%$ & $4.34 \%$ & $49.29 \%$ \\
$\mathbf{2 0 0 5}$ & $6.71 \%$ & $41.75 \%$ & $19.47 \%$ & $78.38 \%$ \\
$\mathbf{2 0 0 6}$ & $10.15 \%$ & $56.14 \%$ & $18.51 \%$ & $111.37 \%$ \\
$\mathbf{2 0 0 7}$ & $3.05 \%$ & $60.90 \%$ & $6.74 \%$ & $125.62 \%$ \\
$\mathbf{2 0 0 8}$ & $1.91 \%$ & $63.98 \%$ & $1.57 \%$ & $129.16 \%$ \\
$\mathbf{2 0 0 9}$ & $7.01 \%$ & $75.47 \%$ & $6.94 \%$ & $145.06 \%$ \\
$\mathbf{2 0 1 0}$ & $9.21 \%$ & $91.63 \%$ & $10.14 \%$ & $169.91 \%$ \\
$\mathbf{2 0 1 1}$ & $5.21 \%$ & $101.62 \%$ & $6.15 \%$ & $186.51 \%$ \\
$\mathbf{2 0 1 2}$ & $-1.64 \%$ & $98.31 \%$ & $1.47 \%$ & $190.72 \%$ \\
$\mathbf{2 0 1 3}$ & $4.95 \%$ & $108.13 \%$ & $6.21 \%$ & $208.78 \%$ \\
\hline & $5.44 \%$ & $119.44 \%$ & $6.95 \%$ & $230.24 \%$ \\
\hline
\end{tabular}

we invested the amount of the DJIA index level (\$7672) in 1997 in our portfolio guided by Graham's formula and purchased securities with RGV's greater than or equal to 1.25 while selling them when below 1, would end up as $\$ 32,861.14$ at the end of June, 2013. If we invested $\$ 7672$ in our portfolio as guided by Graham's formula and purchased securities with an RGV greater than or equal to 1.5 while selling them when below 1, our $\$ 7672$ would be worth $\$ 49453.44$ at the end of June, 2013. Bear in mind that this excludes any transaction costs associated with buying and selling as dictated by Graham's formula or fees associated with holding a mutual/index fund to mimic the DJIA portfolio. This is also under the assumption that our portfolio can only be revisited once per year, meaning we check the RGV level of our securities only on June $30^{\text {th }}$ of each year. This means that our securities could be meeting our sell criterion sometime during the year before or after June $30^{\text {th }}$, and we would not have sold. The opposite case is also true-sometime during the year before or after June $30^{\text {th }}$ the stock could have been meeting our buy criterion but we would not have bought. This represents a very illiquid portfolio which only adjusts its securities based on annual re-evaluation. It would have been interesting to see if Graham's formula could have outperformed or even underperformed the market more so if we allowed for monthly or even daily re-evaluation.

\section{Concluding Remarks}

We find Graham's formula mystifying in ways. One of the most simple valuation methods that we have ever seen produced by one of the most intelligent and distinguished minds in the history of finance. Even though the data that was presented to you suggests that Graham's formula can be actively used to outperform the market given a sufficient margin of safety, we are still hesitant moving forward to use this method as investors because of how worryingly simple it is. The formula does not ask for much and thus you're left feeling that you will not get much in return either. We feel this could be one of the main underlying reasons for the formula's success. No matter the evidence you present in favor of its predictive abilities, it likely is not used much if at all by insti- 
tutional investors because of it perceived naivety. This could give the user of the formula an advantage. The market, a collection of all investors, determines stock prices at any given point in time. If this formula was used on a large scale by many investors the probability of outperforming the market would have to decline as prices reflect Graham's intrinsic value. Given that Graham's formula has been successful in retrospect and its usage is likely limited, this could provide potential for individual investors to do well in the markets. For those of us who do not have enough time to engage in individual security analysis and to keep up with the market, Graham's formula provides hope. Over the past seventeen years, using Graham's formula could have netted an investor more than double, in dollar amounts, what the Dow Jones Industrial Average would have netted them. Historical performance is never an indicator of future performance, but we have compelling evidence to suggest that there is some strength in this formula. Combined with further analysis and multiple other analyses the formula could provide an individual investors appreciable returns and ease of mind.

\section{References}

[1] Arbel, A., Carvell, S. and Postnieks, E. (1988) The Smart Crash of October $19^{\text {th }}$. Harvard Business Review, $124-136$.

[2] Benjamin, G. (2013) Investopedia. http://www.investopedia.com/terms/b/bengraham.asp

[3] Graham, B. (2006) The Intelligent Investor. Harper, New York.

[4] Morningstar (2013) Morningstar Articles RSS. 